\title{
A System of $n=3$ Coupled Oscillators with Magnetic Terms: Symmetries and Integrals of Motion
}

\author{
Manuel F. RAN NADA
}

Departamento de Física Teórica, Facultad de Ciencias, Universidad de Zaragoza, 50009 Zaragoza, Spain

E-mail: mfran@posta.unizar.es

Received July 06, 2005, in final form September 16, 2005; Published online September 20, 2005

Original article is available at http://www.emis.de/journals/SIGMA/2005/Paper004/

\begin{abstract}
The properties of a system of $n=3$ coupled oscillators with linear terms in the velocities (magnetic terms) depending in two parameters are studied. We proved the existence of a bi-Hamiltonian structure arising from a non-symplectic symmetry, as well the existence of master symmetries and additional integrals of motion (weak superintegrability) for certain particular values of the parameters.
\end{abstract}

Key words: non-symplectic symmetries; bi-Hamiltonian structures; master symmetries; cubic integrals

2000 Mathematics Subject Classification: 37J15; 70H06; 70H33

\section{Introduction}

The main objective of this paper is to present a study of a system that, although arising from a quadratic Hamiltonian of simple form, it is however endowed with several interesting properties related with the theory of nonstandard symmetries and the existence of cubic integrals of motion.

Let us consider the Lagrangian of a system of $n=3$ coupled oscillators with an additional coupling term linear in the velocities (the so-called magnetic terms)

$$
\begin{aligned}
& L=\frac{1}{2}\left(v_{1}^{2}+v_{2}^{2}+v_{3}^{2}\right)-\left(V_{1}+V_{0}\right), \\
& V_{0}=\frac{1}{2} k\left(q_{21}^{2}+q_{32}^{2}+q_{13}^{2}\right), \\
& V_{1}=b\left(q_{32} v_{1}+q_{13} v_{2}+q_{21} v_{3}\right),
\end{aligned}
$$

where $b$ and $k$ are arbitrary constants, $q_{i j}$ denote $q_{i}-q_{j}$, and we assume that, for easiness of notation, the mass $m$ of the particle is set equal to unity. See Refs. [6] and [14] for two recent papers dealing with similar systems.

The starting point is to consider $L_{0}=T-V_{0}$, where $T$ denotes the kinetic energy, as the basic original system, and the additional term $V_{1}$ (linear in the velocities) as introducing a deformation. We try to formulate the results in explicit dependence of $b$ in order to study the changes of the dynamics when the parameter $b$ varies.

The Lagrangian $L$ has two exact Noether symmetries generated by the vector fields

$$
\begin{aligned}
Y_{2} & =\frac{\partial}{\partial q_{1}}+\frac{\partial}{\partial q_{2}}+\frac{\partial}{\partial q_{3}}, \\
Y_{3} & =q_{32} \frac{\partial}{\partial q_{1}}+q_{13} \frac{\partial}{\partial q_{2}}+q_{21} \frac{\partial}{\partial q_{3}},
\end{aligned}
$$


with associated constants of the motion

$$
\begin{aligned}
& I_{2}=v_{1}+v_{2}+v_{3}, \\
& I_{3}=q_{32} v_{1}+q_{13} v_{2}+q_{21} v_{3}-b\left(q_{21}^{2}+q_{32}^{2}+q_{13}^{2}\right) .
\end{aligned}
$$

The first constant $I_{1}$ is the total energy given by

$$
I_{1}=\Delta(L)-L=\frac{1}{2}\left(v_{1}^{2}+v_{2}^{2}+v_{3}^{2}\right)+\frac{1}{2} k\left(q_{21}^{2}+q_{32}^{2}+q_{13}^{2}\right) .
$$

where $\Delta=v_{i}\left(\partial / \partial v_{i}\right)$ represents the Liouville vector field.

The Legendre transformation

$$
p_{1}=v_{1}-b q_{32}, \quad p_{2}=v_{2}-b q_{13}, \quad p_{3}=v_{3}-b q_{21}
$$

leads to the following Hamiltonian

$$
\begin{aligned}
H & =\frac{1}{2}\left[\left(p_{1}+b q_{32}\right)^{2}+\left(p_{2}+b q_{13}\right)^{2}+\left(p_{3}+b q_{21}\right)^{2}\right]+\frac{1}{2} k\left(q_{21}^{2}+q_{32}^{2}+q_{13}^{2}\right) \\
& =\frac{1}{2}\left(p_{1}^{2}+p_{2}^{2}+p_{3}^{2}\right)+b\left(L_{1}+L_{2}+L_{3}\right)+\frac{1}{2}\left(b^{2}+k\right)\left(q_{21}^{2}+q_{32}^{2}+q_{13}^{2}\right)
\end{aligned}
$$

and the above three constants become

$$
J_{1}=H, \quad J_{2}=p_{1}+p_{2}+p_{3}, \quad J_{3}=L_{1}+L_{2}+L_{3},
$$

where $L_{i}, i=1,2,3$, denote the components of the angular momentum $L_{i}=q_{j} p_{k}-q_{k} p_{j}$. This three integrals satisfy $\left\{J_{r}, J_{s}\right\}=0, r, s=1,2,3$, and make of $H$ an integrable system for all the values of $b$ and $k$. Thus, we arrive to the following result

Proposition 1. If we consider the magnetic term $V_{1}$ as a continuous deformation of the original system $L_{0}=T-V_{0}$ (the value of $b$ representing the intensity of the deformation) then this deformation preserves both the existence of Noether symmetries and the integrability of the original undeformed system.

Notice that $H$ can also be written as $H=J_{0}+b J_{3}$ with $J_{0}$ defined as follows

$$
J_{0}=\frac{1}{2}\left(p_{1}^{2}+p_{2}^{2}+p_{3}^{2}\right)+\frac{1}{2}\left(b^{2}+k\right)\left(q_{21}^{2}+q_{32}^{2}+q_{13}^{2}\right) .
$$

\section{Symmetries and integrals of motion}

In differential geometric terms, the dynamics of a time-independent Hamiltonian system is determined by a vector field on the $2 n$-dimensional cotangent bundle $T^{*} Q$ of a $n$-dimensional manifold $Q$. Cotangent bundles are manifolds endowed, in a natural or canonical way, with a symplectic structure $\omega_{0}$ that, in coordinates $\left\{\left(q_{j}, p_{j}\right) ; j=1,2, \ldots, n\right\}$, is given by

$$
\omega_{0}=d q_{j} \wedge d p_{j}, \quad \omega_{0}=-d \theta_{0}, \quad \theta_{0}=p_{j} d q_{j}
$$

(we write all the indices as subscripts and we use the summation convention on the repeated index). Given a differentiable function $F=F(q, p)$, the vector field $X_{F}$ defined as the solution of the equation

$$
i\left(X_{F}\right) \omega_{0}=d F
$$

is called the Hamiltonian vector field of the function $F$. There are two important properties:

(i) The Hamiltonian vector field of a given function is well defined without ambiguities. This uniqueness is a consequence of the symplectic character of the two-form $\omega_{0}$.

(ii) Suppose that we are given a Hamiltonian $H=H(q, p)$. Then the dynamics is given by the Hamiltonian vector field $\Gamma_{H}$ of the Hamiltonian function. That is, $i\left(\Gamma_{H}\right) \omega_{0}=d H$. 


\subsection{Bi-Hamiltonian structure}

At this point we recall that a (infinitesimal) dynamical symmetry of a Hamiltonian system $\left(T^{*} Q, \omega_{0}, H\right)$ is a vector field $Y$ such that it satisfies $\left[Y, \Gamma_{H}\right]=0$. On the other hand it is known that, in some very particular cases, the Hamiltonian systems can admit dynamical but non-symplectic symmetries (for a classification of the symmetries in geometric terms see [4] and [15]). In this case we have the following property.

Proposition 2. Suppose there is a vector field $Y$ that is a dynamical symmetry of $\Gamma_{H}$ but does not preserve the symplectic two-form

$$
\mathcal{L}_{Y} \omega_{0}=\omega_{Y} \neq 0 .
$$

Then (i) the dynamical vector field $\Gamma_{H}$ is bi-Hamiltonian, and (ii) the function $Y(H)$ is the new Hamiltonian, and therefore it is a constant of motion.

Proof. For a proof of this proposition see [1, 2, 18], and references therein. A similar property is studied in [20, 22] for the case of Poisson manifolds.

Let us denote by $Y_{3}$ the Hamiltonian vector field of the function $J_{3}$

$$
i\left(Y_{3}\right) \omega_{0}=d J_{3} .
$$

Then we have the following property:

Proposition 3. The vector field $Y_{3}$, defined by (3) as the canonical infinitesimal symmetry associated with $J_{3}$, can be written as a linear combination of two dynamical but non-symplectic symmetries of $\Gamma_{H}$.

Proof. We can write $Y_{3}$ as follows

$$
\begin{aligned}
& Y_{3}=Y_{3}^{a}-Y_{3}^{b}, \\
& Y_{3}^{a}=q_{3} \frac{\partial}{\partial q_{1}}+q_{1} \frac{\partial}{\partial q_{2}}+q_{2} \frac{\partial}{\partial q_{3}}+p_{3} \frac{\partial}{\partial p_{1}}+p_{1} \frac{\partial}{\partial p_{2}}+p_{2} \frac{\partial}{\partial p_{3}}, \\
& Y_{3}^{b}=q_{2} \frac{\partial}{\partial q_{1}}+q_{3} \frac{\partial}{\partial q_{2}}+q_{1} \frac{\partial}{\partial q_{3}}+p_{2} \frac{\partial}{\partial p_{1}}+p_{3} \frac{\partial}{\partial p_{2}}+p_{1} \frac{\partial}{\partial p_{3}} .
\end{aligned}
$$

Then the vector field $Y_{3}^{a}$ (or $Y_{3}^{b}$ ) is neither locally-Hamiltonian with respect to $\omega_{0}$

$$
\mathcal{L}_{Y_{3}^{a} \omega_{0}}=\omega_{a} \neq 0
$$

nor a symmetry of the Hamiltonian function, $Y_{3}^{a}(H) \neq 0$. Nevertheless it satisfies

$$
\left[Y_{3}^{a}, \Gamma_{H}\right]=0 .
$$

Therefore $Y_{3}^{a}$ (or $Y_{3}^{b}$ ) is a dynamical but non-symplectic symmetry of the Hamiltonian system.

Thus the dynamical vector field $\Gamma_{H}$ is a bi-Hamiltonian system with respect to $\left(\omega_{0}, \omega_{a}\right)$

$$
i\left(\Gamma_{H}\right) \omega_{0}=d H, \quad i\left(\Gamma_{H}\right) \omega_{a}=d H_{a},
$$

with $\omega_{a}$ and $H_{a}=Y_{a}(H)$ given by

$$
\begin{aligned}
& \omega_{a}=d q_{1} \wedge\left(d p_{2}+d p_{3}\right)+d q_{2} \wedge\left(d p_{1}+d p_{3}\right)+d q_{3} \wedge\left(d p_{1}+d p_{3}\right), \\
& H_{a}=\frac{1}{2} J_{2}^{2}-H .
\end{aligned}
$$

The function $H_{a}=Y_{a}(H)$ can be considered as a new Hamiltonian for $\Gamma_{H}$. 
The bi-Hamiltonian structure $\left(\Gamma_{H}, \omega_{0}, \omega_{a}\right)$ determines a $(1,1)$-tensor field $R$ by the relation

$$
\omega_{a}(X, Y)=\omega_{0}(R X, Y), \quad \forall X, Y \in \mathcal{X}\left(T^{*} Q\right),
$$

or, equivalently, $R=\widehat{\omega}_{0}^{-1} \circ \widehat{\omega}_{a}\left[\right.$ with $\left.\widehat{\omega}(X)=i(X) \omega \in \wedge^{1}\left(T^{*} Q\right)\right]$. We have obtained

$$
\begin{aligned}
R= & \frac{\partial}{\partial q_{1}} \otimes\left(d q_{2}+d q_{3}\right)+\frac{\partial}{\partial q_{2}} \otimes\left(d q_{1}+d q_{3}\right)+\frac{\partial}{\partial q_{3}} \otimes\left(d q_{1}+d q_{2}\right) \\
& +\frac{\partial}{\partial p_{1}} \otimes\left(d p_{2}+d p_{3}\right)+\frac{\partial}{\partial p_{2}} \otimes\left(d p_{1}+d p_{3}\right)+\frac{\partial}{\partial p_{3}} \otimes\left(d p_{1}+d p_{2}\right) .
\end{aligned}
$$

Starting with the basic Hamiltonian system $\left(\omega_{0}, \Gamma_{0}=\Gamma_{H}, d H_{0}=d H\right)$, and iterating $R$, we can construct a sequence of 2 -forms $\omega_{k}$, vector fields $\Gamma_{k}$, and exact 1 -forms $d H_{k}, k=1,2, \ldots$, defined by $\widehat{\omega}_{k}=\widehat{\omega}_{0} \circ R^{k}, \Gamma_{k}=R^{k}\left(\Gamma_{0}\right)$, and $d H_{k}=R^{k}\left(d H_{0}\right)$, that leads to sequence of biHamiltonian vector fields. The two first steps become

$$
i\left(\Gamma_{0}\right) \omega_{1}=i\left(\Gamma_{1}\right) \omega_{0}=d H_{1}, \quad i\left(\Gamma_{0}\right) \omega_{2}=i\left(\Gamma_{1}\right) \omega_{1}=i\left(\Gamma_{2}\right) \omega_{0}=d H_{2},
$$

where $\omega_{2}, \Gamma_{2}$, and $H_{2}$, are given by

$$
\omega_{2}=2 \omega_{0}+\omega_{1}, \quad \Gamma_{2}=2 \Gamma_{0}+\Gamma_{1}, \quad H_{2}=2 H_{0}+H_{1} .
$$

So, in this particular case, the sequence $\left(\omega_{k}, \Gamma_{k}, d H_{k}\right)$ closes over itself after two steps.

\subsection{Master symmetries}

The function $T=T(q, p)$ is said to be a generator of constants of motion of degree $m$ if it is not preserved by the dynamics but it generates an integral of motion by time derivation

$$
\frac{d}{d t} T \neq 0, \quad \ldots, \quad \frac{d^{m-1}}{d t^{m-1}} T \neq 0, \quad \frac{d^{m}}{d t^{m}} T=0 .
$$

In differential geometric terms, a vector field $X$ on $T^{*} Q$ that satisfies

$$
\left[\Gamma_{H}, X\right] \neq 0, \quad\left[\Gamma_{H}, \widetilde{X}\right]=0, \quad \widetilde{X}=\left[\Gamma_{H}, X\right]
$$

is called a "master symmetry" or a "generator of symmetries" of degree $m=1$ for $\Gamma_{H}[3,5,7,17]$. If a Hamiltonian $H$ admits two of such generators, $T_{1}, T_{2}$, (master integrals) then the function $I_{12}$ defined by

$$
I_{12}=I_{2} T_{1}-I_{1} T_{2}, \quad I_{r}=\frac{d}{d t} T_{r}, \quad r=1,2,
$$

is a new constant of motion (independent of $I_{1}$ and $I_{2}$ ). In geometric terms, if we denote by $X_{r}$, the Hamiltonian vector fields of $T_{r}$, then $\widetilde{X}_{r}$ is Hamiltonian as well, and we have

$$
i\left(X_{r}\right) \omega_{0}=d T_{r}, \quad i\left(\widetilde{X}_{r}\right) \omega_{0}=d \widetilde{T}_{r}, \quad \widetilde{T}_{r}=\Gamma_{H}\left(T_{r}\right), \quad r=1,2,
$$

so that the vector field $X_{12}$ defined as

$$
X_{12}=T_{1} \widetilde{X}_{2}-T_{2} \widetilde{X}_{1}+\widetilde{T}_{2} X_{1}-\widetilde{T}_{1} X_{2}, \quad i\left(X_{12}\right) \omega_{0}=d I_{12},
$$

is a symmetry of the system $\left(T^{*} Q, \omega_{0}, H\right)$ with the function $I_{12}$ as its associated constant of motion. 
The Hamiltonian vector field $X_{1}$ of the function $T_{1}=q_{1}+q_{2}+q_{3}$ satisfies

$$
i\left(X_{1}\right) \omega_{0}=d T_{1}, \quad\left[\Gamma_{H}, X_{1}\right]=\widetilde{X}_{1}, \quad \widetilde{X}_{1}=\frac{\partial}{\partial q_{1}}+\frac{\partial}{\partial q_{2}}+\frac{\partial}{\partial q_{3}}, \quad\left[\Gamma_{H}, \widetilde{X}_{1}\right]=0,
$$

so $X_{1}$ is a "generator of symmetries" of degree $m=1$ for (2). Similarly if we consider $T_{2}=$ $q_{1} p_{1}+q_{2} p_{2}+q_{3} p_{3}$ we obtain

$$
i\left(X_{2}\right) \omega_{0}=d T_{2}, \quad\left[\Gamma_{H}, X_{2}\right]=\widetilde{X}_{2}, \quad\left[\Gamma_{H}, \widetilde{X}_{2}\right] \stackrel{*}{=} 0,
$$

where the asterisk means that the Lie bracket vanishes only if $k+b^{2}=0$. Hence in the particular case $k+b^{2}=0$, the system is superintegrable with an additional integral of the form $I_{12}$ that becomes

$$
J_{4} \equiv I_{12}=L_{1}\left(p_{2}-p_{3}\right)+L_{2}\left(p_{3}-p_{1}\right)+L_{3}\left(p_{1}-p_{2}\right) .
$$

\subsection{Third-order integrals}

Linear integrals of motion be obtained from exact Noether symmetries and quadratic integrals from Hamilton-Jacobi (Schrödinger) separability but third-order integrals must be obtaining by other alternative procedures; because of this, the number of known integrable systems admitting cubic in the momenta constants is very limited [9, 10, 13, 21, 23]. For $n=2$ we can point out the Fokas-Lagerstrom and the Holt potentials for the Euclidean plane [8, 12], and three of the Drach potentials for the pseudoeuclidean plane [16, 19]. For $n=3$ two very special systems are well known: the three-particle Toda chain and three-particle Calogero-Moser system which are both endowed with a cubic constant of the form

$$
J=\frac{1}{3}\left(p_{1}^{3}+p_{2}^{3}+p_{3}^{3}\right)+\text { terms of first order. }
$$

At this point we recall the following property: polynomial constants of the motion for mechanical systems (Riemmanian metric with a potential) are either even or odd in the momenta (for a discussion of this result see, e.g., [11]). Therefore if $J$ is a cubic integral for a Hamiltonian such as the Calogero-Moser or the Toda systems then it must also contain linear terms but neither quadratic nor independent ones. Nevertheless this property is not true for the more general case of a non-mechanical Lagrangian. In our case, due to the presence of linear terms in the velocities (magnetic terms) when looking for third-order integrals we must assume for the function $J_{4}$ a polynomial expression containing also even powers.

We start with a third-order function $J$ of a general form

$$
J=\sum_{i+j+k=3} a_{i j k} p_{1}^{i} p_{2}^{j} p_{3}^{k}+\sum_{i+j+k=2} b_{i j k} p_{1}^{i} p_{2}^{j} p_{3}^{k}+\sum_{i} c_{i} p_{i}+d,
$$

where $a_{i j k}, b_{i j k}, c_{i}$, and $d$ are functions of $q_{1}, q_{2}, q_{3}$. Then the condition $\{H, J\}=0$ leads to a system of equations; some of these equations restrict the expressions of the coefficients $a_{i j k}$, $i+j+k=3$, of the higher-order terms; the other equations couple the derivatives of $V_{1}$ and $V_{0}$ with the coefficients $a_{i j k}, b_{i j k}, c_{i}$, and $d$ or with the derivatives of these coefficients. Unfortunately, the general case is difficult to be studied but some particular cases can be analyzed assuming simple particular forms for the coefficients $a_{i j k}$.

We have studied the existence of a cubic integral of such a particular form for this threeparticle system and we have arrived (we omit the details) at the existence of the following function

$$
\begin{aligned}
J_{4}= & \frac{1}{3}\left(p_{1}^{3}+p_{2}^{3}+p_{3}^{3}\right)-b\left(q_{32} p_{1}^{2}+q_{13} p_{2}^{2}+q_{21} p_{3}^{2}\right) \\
& +b^{2}\left(z_{1} p_{1}+z_{2} p_{2}+z_{3} p_{3}\right)+9 b^{3}\left(q_{21}^{3}+q_{13}^{3}+q_{32}^{3}\right),
\end{aligned}
$$


where $z_{1}, z_{2}$, and $z_{3}$, are given by

$$
z_{1}=4\left(q_{21}^{2}+q_{13}^{2}\right)+q_{32}^{2}, \quad z_{2}=4\left(q_{32}^{2}+q_{21}^{2}\right)+q_{13}^{2}, \quad z_{3}=4\left(q_{13}^{2}+q_{32}^{2}\right)+q_{21}^{2} .
$$

The important point is that the function $J_{4}$ is a constant of motion, not for all the values of the parameters $(b, k)$, but only in the particular case of $k$ and $b$ satisfying $k=8 b^{2}$. We have verified that in this case the four functions $H, J_{2}, J_{3}, J_{4}$ are independent and that the system $\left(J_{2}, H, J_{4}\right)$ is involutive (nevertheless $\left\{J_{3}, J_{4}\right\} \neq 0$ ). Thus, when $k$ and $b$ are related by $k=8 b^{2}$ then the system becomes superintegrable with an additional third-order integral.

We finally note that $J_{2}$ is linear, $H$ quadratic, and $J_{4}$ cubic, and that they have expressions of the form

$$
J_{2}=p_{1}+p_{2}+p_{3}, \quad H=p_{1}^{2}+p_{2}^{2}+p_{3}^{2}+\cdots, \quad J_{4}=p_{1}^{3}+p_{2}^{3}+p_{3}^{3}+\cdots
$$

that closely resemble the expressions of the three first constants of Toda or the Calogero-Moser systems.

\section{Final comments}

The Lagrangian system (1) (Hamiltonian (2)) is a system endowed with a bi-Hamiltonian structure, non-symplectic symmetries and master symmetries. Moreover for a particular value of the parameter $b$, it becomes superintegrable with a cubic integral.

The non-symplectic symmetry $Y_{3}^{a}$ is $b$-independent and hence the bi-Hamiltonian structure $\left(\Gamma_{H}, \omega_{0}, \omega_{a}\right)$ is the one endowed by the the original oscillators system $L_{0}=T-V_{0}$ that is preserved under the $b$-deformation introduced by the magnetic term $V_{1}$. Concerning the master symmetries, the first one is always present but the second only exists if $k+b^{2}=0$. So in this particular case the system becomes (weakly) superintegrable. This particular case is very peculiar because the Hamiltonian (2) becomes a system of $n=3$ particles with velocity-linear couplings but without the potential term.

Finally the particular $k=8 b^{2}$ case is very interesting. First because, as stated above, the number of known integrable systems with third-order integrals is very small. Second because the existence of this property for a very particular case of the parameters resembles situations characterizing other known systems (e.g., the Hénon-Heiles system is only integrable for some very particular values of a parameter). Finally because the expression obtained for $J_{4}$ in (8) shows similarity with the third-order integral (7) of the Toda or the Calogero-Moser systems. This leads to the necessity of the study of the more general $n>3$ case, that poses the question of the nature of the interaction (in the Toda chain the interactions are between neighbours but in the Calogero-Moser case they are between every two particles). On the other hand this also leads to the search of a Lax pair generating the three functions $\left(J_{2}, H, J_{4}\right)$, as traces of the powers of an appropriate matrix. Nevertheless, notice that this Lax structure (if it exists) must

correspond to the $L$ system with the additional condition $k=8 b^{2}$; so it cannot be obtained as a continuous deformation of a previous Lax pair for the $L_{0}$ system. We think that these are open questions that must be investigated.

\section{Acknowledgements}

Support of projects BFM-2003-02532 and FPA-2003-02948 is acknowledged. 
[1] Cariñena J.F., Ibort L.A., Non-Noether constants of motion, J. Phys. A: Math. Gen., 1983, V.16, 1-7.

[2] Cariñena J.F., Marmo G., Rañada M.F., Non-symplectic symmetries and bi-Hamiltonian structures of the rational harmonic oscillator, J. Phys. A: Math. Gen., 2002, V.35, L679-L686.

[3] Caseiro R., Master integrals, superintegrability and quadratic algebras, Bull. Sci. Math., 2002, V.126, 617630 .

[4] Crampin M., Tangent bundle geometry for Lagrangian dynamics, J. Phys. A: Math. Gen., 1983, V.16, $3755-3772$.

[5] Damianou P.A., Symmetries of Toda equations, J. Phys. A, 1993, V.26, 3791-3796.

[6] Damianou P.A., Sophocleous C., Lie point symmetries of Hamiltonian systems, Bull. Greek Math. Soc., 2000, V.44, 87-96.

[7] Fernandes R.L., On the master symmetries and bi-Hamiltonian structure of the Toda lattice, J. Phys. A: Math. Gen., 1993, V.26, 3797-3803.

[8] Fokas A.S., Lagerstrom P.A., Quadratic and cubic invariants in classical mechanics, J. Math. Anal. Appl. 1980, V.74, 325-341.

[9] Gravel S., Hamiltonians separable in Cartesian coordinates and third-order integrals of motion, J. Math. Phys., 2004, V.45, 1003-1019.

[10] Gravel S., Winternitz P., Superintegrability with third-order integrals in quantum and classical mechanics, J. Math. Phys., 2002, V.43, 5902-5912.

[11] Hietarinta J., Direct methods for the search of the second invariant, Phys. Rep., 1987, V.147, 87-154.

[12] Holt C.R., Construction of new integrable Hamiltonians in two degrees of freedom, J. Math. Phys., 1982, V.23, 1037-46.

[13] McLenaghan R.G., Smirnov R.G., The D., Towards a classification of cubic integrals of motion, in Proceedings of the First International Workshop "Superintegrability in Classical and Quantum Systems" (September 16-21, 2002, Montreal), Editors P. Tempesta et al., CRM Proc. Lecture Notes, V.37, Providence, RI, Amer. Math. Soc., 2004, 199-209.

[14] McSween E., Winternitz P., Integrable and superintegrable Hamiltonian systems in magnetic fields, J. Math. Phys., 2000, V.41, 2957-2967.

[15] Prince G., Toward a classification of dynamical symmetries in classical mechanics, Bull. Austral. Math. Soc., 1983, V.27, 53-71.

[16] Rañada M.F., Superintegrable $n=2$ systems, quadratic constants of motion, and potentials of Drach, J. Math. Phys., 1997, V.38, 4165-4178.

[17] Rañada M.F., Superintegrability of the Calogero-Moser system: constants of motion, master symmetries, and time-dependent symmetries, J. Math. Phys., 1999, V.40, 236-247.

[18] Rañada M.F., Dynamical symmetries, bi-Hamiltonian structures, and superintegrable $n=2$ systems, J. Math. Phys., 2000, V.41, 2121-2134.

[19] Rañada M.F., Santander M., Complex Euclidean super-integrable potentials, potentials of Drach, and potential of Holt, Phys. Lett. A, 2001, V.278, 271-279.

[20] Sergyeyev A., A simple way of making a Hamiltonian system into a bi-Hamiltonian one, Acta Appl. Math., 2004, V.83, 183-197.

[21] Sheftel M., On the classification of third-order integrals of motion in two-dimensional quantum mechanics, in Proceedings of the First International Workshop "Superintegrability in Classical and Quantum Systems" (September 16-21, 2002, Montreal), Editors P. Tempesta et al., CRM Proc. Lecture Notes, V.37, Providence, RI, Amer. Math. Soc., 2004, 187-197.

[22] Smirnov R.G., Bi-Hamiltonian formalism: a constructive approach, Lett. Math. Phys., 1997, V.41, 333-347.

[23] Thompson G., Polynomial constants of motion in flat space, J. Math. Phys., 1984, V.25, 3474-3478. 